Case Report,

\title{
One-Year Follow-Up of Isolated Trapezoid Fracture from Occult Trauma: A Case Report
}

\author{
Sitthiphong suwannaphisit ${ }^{1}$, Porames Suwanno ${ }^{2}$, Boonsin Tangtrakulwanich ${ }^{3}$ \\ 1,2,3 Department of Orthopedics, Faculty of Medicine, Prince of Songkla University, Songkhla, Thailand
}

\begin{abstract}
:
Background

Herein we present a rare case of isolated trapezoid fracture from occult trauma and review this type of fracture as related to sports injuries. The common presentation of occult trauma is important and proper imaging will lead to accurate diagnosis and consequent treatment with excellent results.
\end{abstract}

\section{Case presentation}

A 28-year-old right hand-dominant female presented with pain in her right hand for 1 week following a boxing class in fitness training. She was diagnosed as isolated trapezoid fracture. She was successfully treated with short arm cast immobilization for 3 weeks and a moveable wrist splint for 3 weeks with activity modification.

A one year follow up confirmed excellent clinical results.

\section{Conclusion}

Isolated sports-related trapezoid fractures are rare and the diagnosis is usually missed. Advanced imaging can be helpful. Following conservative treatment our patient was able to return to normal activities and continued to have no problems at the 1 year follow up.

Key words: Trapezoid fracture, Sport-related injury, Treatment outcomes

\section{Background:}

Isolated trapezoid fracture is the least common carpal bone fracture, accounting for only $0.4 \%$ of these fractures [1]. High energy axial loading or bending is the common mechanisms of injury of the isolated trapezoidfracture [2]. Due to its anatomy and geography, surrounded by intercarpal ligaments, other carpal bones, and at the base of the second metacarpal, it is the most secure carpal bone [3]. Only a few studies have been published on the isolated carpal bone fracture.

\section{Presentation of case:}

The patient was a 28-year-old right hand-dominant female who presented to the hand clinic with pain in her right hand for 1 week following a boxing class in fitness training. She was an OB-GYN doctor and her hobby was Muay-Thai boxing, which she practiced three times per week. On the date of her injury, she complained of pain and swelling in her right hand after finishing her MuayThai class. She went to the ER where PA and oblique radiographic views were taken (Figure 1), which were, however, unremarkable. She was given an NSAID but her pain and swelling did not improve. She was suspicious that she may have suffered an occult fracture or ligamentous injury around her wrist, and decided by herself to order an MRI of her right hand, she then showed the MRI to an orthopedist at the Orthopedics OPD, who identified a transvere fracture at the trapezoid with surrounding marrow edema with slight subluxation of the scapho-trapezoid joint, and also a questionable sclerotic line with marrow edema at the scaphoid waist (Figure 2). A second physical examination found tenderness on the dorsum of the second metacarpal bone just proximal to the base, but without tenderness at the scaphoid tubercle or anatomical snuffbox. An axial loading test was also negaitve. An orthopedist discussed our 
findings with the patient and finally she decided on conservative treatment by immobilization. A short arm cast was placed around her wrist and the analgesic and anti-inflammatory medications continued. However, after 3 weeks of treatment, she returned to our clinic and ask to have the cast removed because she felt she could not tolerate the limitations on her activities and work. She accepted the risk of possible future complications such as further displacement or non-union of the bones, and we applied a moveable wrist splint and counsealed her to limit her activities as much as possible Six weeks later the wrist splint was removed, and she began physical therapy. Two months after the initial injury, she had regained complete and painless mobility of the wrist, without any edema or tenderness, and 3 months after the injury, she had returned to work with no limitations and was performing intense physical exercise using the affected hand without problems, including returning to her Muay-Thai martial arts practice without pain in her hand. A CT scan was done at 3 months, and showed complete bone healing without any subluxation (Figure 3). She said she was very satisfied with the results of the treatment at the last follow up at 1 year with equal grip strength and pinch strength on both sides.

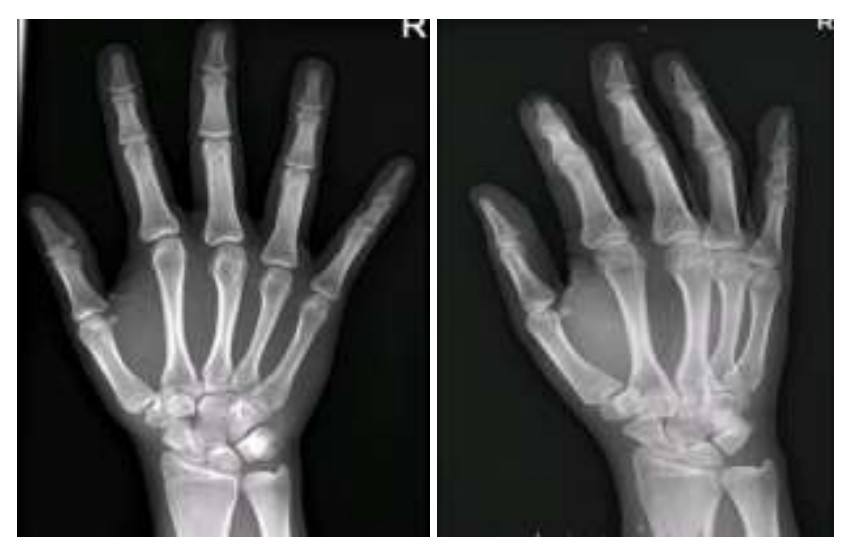

Figure 1 Plain radiograph in AP and oblique views showing unremarkable studies.
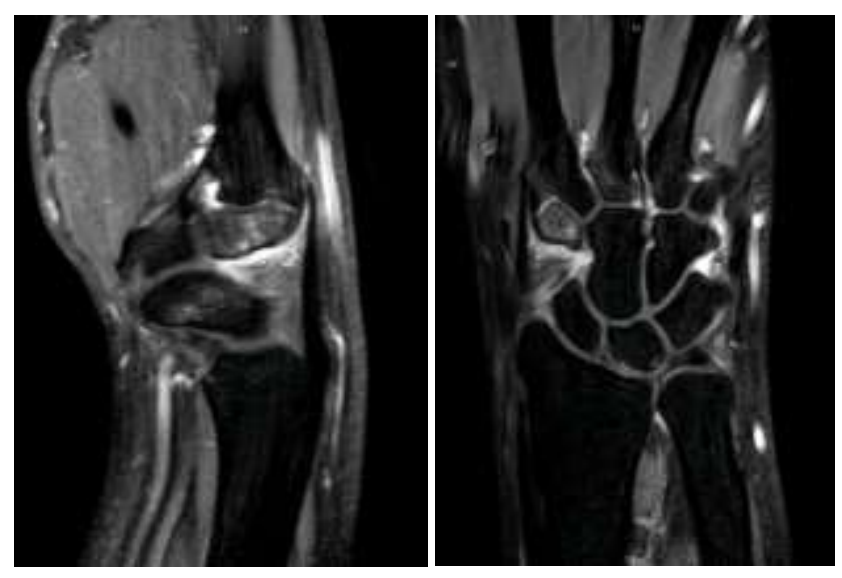

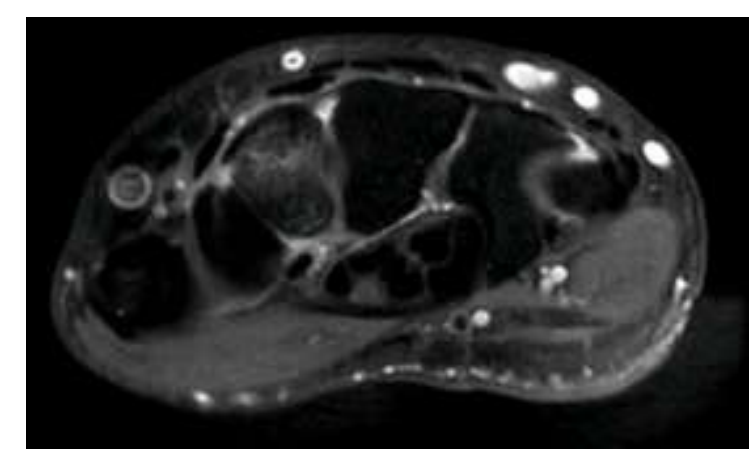

Figure 2 MRI showing a transvere fracture at the trapezoid with surrounding marrow edema with slight subluxation of the scapho-trapezoid joint, and also a questionable sclerotic line with marrow edema at the scaphoid waist.
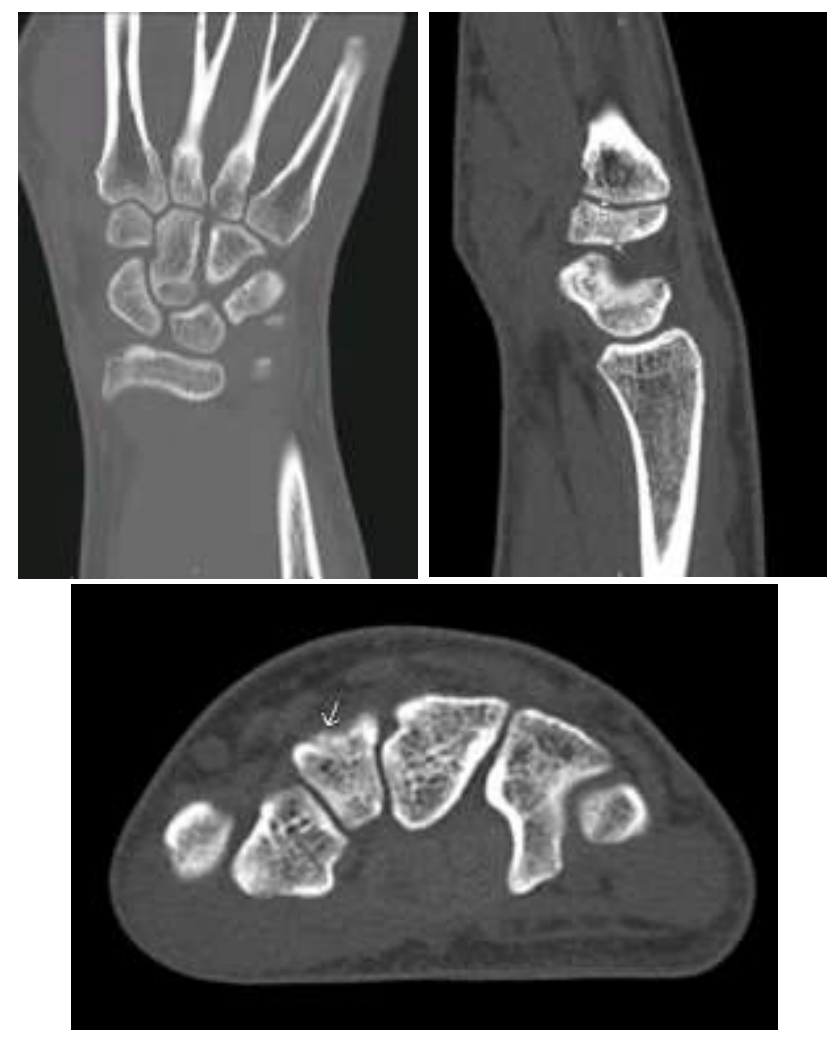

Figure 3 CT scan showing complete bone healing without any subluxation.

\section{Discussion:}

The least common fracture of the carpal bones is the trapezoid fracture with $<1 \%$ of all carpal bone fractures [2]. One study [4] investigated the prevalence of carpal bone fractures in Singapore and found no trapezoid fractures in their series (149 patients with 162 carpal fractures). However, this prevalence may be underestimated due to underdiagnosing of this type of fracture.

Pain or tenderness around the second metacarpal base as well as wrist edema with limited mobility, symptoms which were found in our case, is the common clinical presentation for a trapezoid fracture [5].There are various mechanisms leading to this type of injury which have been noted in 
published studies, notably fist fights [6], motorcycle accidents [7], falls [3], and sportsrelated injuries, especially tennis [5] and soccer [8]. One report involved a male engineer taking boxing lesson, similar to our case [6]. The proper investigations are important in correctly diagnosing these fractures. Although trapezoid fractures are often missed(9), standard antero-posterior and lateral radiographs of the hand are the mainstay first-line imaging, followed by ultrasonography [10], although US can be problematic as it is operator dependent. In difficult cases, MRI, technetium bone scans or CT is recommend [9]. A recent updated study [11] reported 4 cases of isolated trapezoid fractures diagnosed by MRI (initial work-up negative with radiography and one case with CT), and concluded that MRI had advantages over CT, especially in cases of suspected occult fracture [11]. However, our case could diagnose with CT scan. The treatment options vary from conservative management to open reduction and internal fixation, percutaneous fixation, or excision of the fragment $[8,9]$. Conservative treatment with short arm cast immobilization is the gold standard for non-displaced or minimally displaced $(<2 \mathrm{~mm})$ isolated trapezoid fractures with different durations of casting from 4 to 8 weeks [2,3,5-7]. In our case we applied a full cast for 3 weeks and a splint for 3 weeks, which provided excellent results at the 1 year follow up.

\section{Conclusion:}

Isolated sports-related trapezoid fractures are rare and the diagnosis is usually missed. Advanced imaging can be helpful. Following conservative treatment our patient was able to return to normal activities and continued to have no problems at the 1 year follow up.

\section{References:}

[1.] Papp S. Carpal Bone Fractures. Orthop Clin North Am. 2007 Apr; 38(2):251-60.

[2.] Wolfe SW, Hotchkiss RN, Pederson WC, Kozin SH, Cohen MS, editors. Green's operative hand surgery. Seventh edition. Philadelphia, PA: Elsevier; 2017.

[3.] Afifi N, Lu J. A Rare Isolated Trapezoid Fracture. West J Emerg Med. 2011; 12(4):523-4.

[4.] Dennis HHW, Sze ACK, Murphy D. Prevalence of Carpal Fracture in Singapore. J Hand Surg. 2011 Feb; 36(2):278-83.

[5.] Blomquist GA, Hunt III TR, Lopez-Ben RR. Isolated fractures of the trapezoid as a sports injury. Skeletal Radiol. 2013 May; 42(5):735-9.

[6.] Ribeiro LM, Botton MA. Isolated Trapezoid Fracture in a Boxer. Am J Case Rep. 2019 Jun 5;20:790-3.

[7.] Yasuwaki Y, Nagata Y, Yamamoto T, Nakano A, Kikuchi H, Tanaka S. Fracture of the trapezoid bone: A case report. J Hand Surg. 1994 May;19(3):457-9.

[8.] Yamamoto $\mathrm{T}$, Matsushita $\mathrm{T}$, Ito $\mathrm{K}$, Matsushima S, Yoshida K, Kuroda R. Trapezoid Fracture Associated with Scaphoid Fracture in a Football Goalkeeper. Case Rep Orthop. 2019 Sep 5;2019:1-4.

[9.] Vigler M, Aviles A, Lee SK. Carpal Fractures Excluding the Scaphoid. Hand Clin. 2006 Nov; 22(4):501-16.

[10.] Ault DL, Jokerst AR, Kettner NW. Occult isolated fracture of the trapezoid diagnosed by ultrasonography. J Ultrasound [Internet]. 2018 Jul 14 [cited 2020 Jun 7]; Available from:

http://link.springer.com/10.1007/s40477018-0308-3

[11.] Hidlay DT, Levine S. Isolated trapezoid fracture: four cases of a rare fracture on MRI. Radiol Case Rep. 2020 Jun;15(6):769-74. 